PROCESOS

Revista Ecuatoriana de Historia

\title{
Cristiane Alves Camacho dos Santos. Escrevendo a história do futuro. A LEITURA DO PASSADO NO PROCESSO DE INDEPENDÊNCIA DO BRASIL. SÃo Paulo: Alameda, 2017, 236 PP.
}

\section{DOI: http: / / dx.doi.org/10.29078/rp.v0i47.680}

En las últimas décadas, los trabajos sobre la independencia de Brasil han renovado la historiografía de manera novedosa, trayendo nuevas interpretaciones y explicaciones acerca de ese proceso histórico. En el mundo hispanoamericano y, en general, en la historiografía mundial tal proceso es visto como una excepción, ya que allí se logró concretar la independencia y la formación del Estado nacional, supuestamente, sin guerras sangrientas y con un proyecto monárquico en contravía de los nacientes proyectos republicanos que se fueron formando cerca de sus fronteras. Sin embargo, precisamente, eso es lo que la reciente historiografía ha venido desmintiendo: en Brasil igualmente hubo guerras sangrientas y la posibilidad de formar una república estuvo presente. Como muestran diversas investigaciones, los procesos revolucionario e independentista, así como la formación del Estado y la nación brasileños están bastante relacionados con los demás procesos que se empezaron a constituir en el mundo atlántico desde finales del siglo XVIII y durante el XIX.

El libro aquí reseñado es producto de la investigación que Cristiane Santos realizó en su trabajo de maestría en la Universidad de San Pablo, defendida en 2010. A partir de la propuesta metodológica de R. Koselleck sobre las nociones de "espacio de experiencia" y de "horizonte de expectativas" traza su marco de análisis sobre las lecturas que hicieron los actores políticos del período independentista respecto a la narrativa histórica del pasado y los pronósticos del futuro. Así, uno de los principales objetivos es analizar cómo las élites (políticas, económicas y sociales) fueron legitimando en sus discursos entre 1821 y 1822 la independencia de Brasil frente a Portugal. Para ello, principalmente, va a valerse de la prensa y folletos editados en ese momento específico. A partir de esas fuentes primarias examina cuáles eran las interpretaciones y explicaciones de la experiencia histórica que los actores 
políticos de ese momento hacían acerca de su identidad política, así como sus intentos de construir una nueva narrativa histórica de Brasil en la cual su relación con Portugal ya no era de unidad, sino de oposición. De ahí el título de "escribiendo la historia del futuro", pues uno de los objetivo de los actores políticos era crear una historia singular de Brasil. Como señala Santos, Brasil a finales del siglo XVIII y comienzos del XIX no existía como un Estado o como una nación, lo que tenemos es una identidad colectiva asociada a la nación portuguesa. No obstante, esa identidad política empezaría a ser criticada con la revolución pernambucana en 1817 y después sistemáticamente desde 1820 con la Revolución liberal en Portugal. Las medidas tomadas en las cortes de Lisboa crearon diversos conflictos políticos, económicos y sociales entre las élites provinciales lusoamericanas, las cuales divergían entre apoyar o no el nuevo proyecto monárquico impuesto desde la metrópoli. Algunas de las medidas que causaron polémica fueron la extinción del Reino de Brasil, creado en 1815, la exigencia al rey D. Juan VI, que estaba en Río de Janeiro, para que volviera a Portugal, más adelante lo mismo se le exigiría a su hijo, el regente D. Pedro I. Esto, así como la desigualdad en la representación posibilitaba aún más que los lusoamericanos proyectasen su independencia de Portugal.

El libro está dividido en tres capítulos y un epílogo. En el primer capítulo, titulado "Identidades políticas y experiencia del tiempo en la crisis del Antiguo Régimen portugués", analiza cómo se representaba en la narrativa histórica a la América portuguesa, desde mediados del siglo XVIII hasta 1820. Va a mostrar que en un primer momento la representación de esa experiencia histórica mantiene una unidad y se muestra favorable a los hechos de los portugueses en el proceso de colonización. Si bien a finales del siglo XVIII y a comienzos del XIX ya hay una noción de Brasil como una entidad singular, esta está siempre vinculada al imperio portugués. Con la invasión francesa a la península ibérica y el traslado de la Corona a sus territorios en América, la identidad política de los lusoamericanos tendrá significativos cambios cuando la monarquía portuguesa se establece en 1808 en Río de Janeiro, pues ahora Brasil cambiaba su estatus de colonia a ser sede del imperio (44 y ss.) Aquí la autora explica detalladamente algunas de las disputas que hay en la definición en torno del concepto colonización y sus derivaciones (60 y ss.).

En el segundo capítulo, titulado "La tematización de la presencia portuguesa en la América de 1821", examina las narrativas acerca del papel de los portugueses en América. Como se observa, algunas veces dicho papel es presentado positivamente, pues se muestra como articulador de unidad, progreso y civilización; en otras negativamente, ya que es asociado a opresión y explotación. Ahora bien, para explicar ese tema expone la importancia de la apertura del espacio de opinión pública, señala que a partir de 1820 
hay un aumento significativo de publicaciones de periódicos y folletos, que son las fuentes primarias más importantes que va a usar para demostrar sus hipótesis a través de la obra. Aunque, como sabemos, la mayoría de los periódicos fueron publicados en Río de Janeiro, hay un esfuerzo notable por consultar los que se publicaron en otras provincias (como Bahía, Pernambuco, Pará o fuera de Brasil, como el Correio Braziliense publicado en Londres). Esto le permite contrastar diversos discursos e intereses políticos entre las élites provinciales. Podemos observar que en un primer momento gran parte defiende en sus discursos la unidad del imperio, pero estos comenzarían a alternarse con versiones negativas de la colonización, como una forma de opresión (87 y ss.). De esta manera se pueden encontrar ambos discursos al tiempo, según los intereses de los actores políticos en los debates. Lo anterior claramente está ligado al contexto de la Revolución liberal de Porto de 1820 que posibilitaba críticas al imperio y su forma de gobernar. Además, para los lusoamericanos, los acontecimientos en la América española y en la misma Europa servían como ejemplos y referencias para sus propias experiencias y expectativas.

En el tercer capítulo, titulado "La victoria de los 'tres siglos de opresión' y la valorización de las experiencias históricas recientes", analiza cuáles son los discursos históricos expuestos en la prensa, así como las lecturas que se hacían acerca de la colonización portuguesa en América, principalmente a partir de 1821 y 1822. En este momento se refuerza el discurso respecto a los "tres siglos de opresión", que era instrumentalizado para mostrar la incompatibilidad de la historia de Brasil con la de Portugal, en otras palabras, el proyecto político de la unidad de la nación portuguesa esbozado desde la metrópoli era incompatible con el proyecto de emancipación que se comenzaba a expresar en Brasil. Para ello, como muestra la autora, los publicistas lusoamericanos se valieron de las tesis de los abades Raynal y De Pradt, expuestas en sus libros, tanto para legitimar sus discursos acerca de la independencia como para esbozar nuevas construcciones sobre la narrativa histórica de Brasil, por cierto cada vez más politizada.

Finalmente, en el epílogo trata el tema de la cuestión nacional, para ello examina básicamente los trabajos de E. Hobsbawm y A. Smith, quienes abordan el asunto desde diferentes perspectivas. Para el primero, la nación y el nacionalismo toman forma desde el Estado, mientras que para el segundo es desde los grupos o comunidades étnicos que se constituyen. Este análisis final es pertinente porque la investigación de la autora es atravesada por la reflexión sobre formación del Estado y la nación brasileños. Así, muestra que las lecturas que los actores políticos hacían del pasado en el período independentista, de una u otra manera, van a servir como fuente a la historiografía que se empieza a consolidar a mediados del siglo XIX, para legitimar 
la formación del Estado nacional brasileño. Esa naciente historiografía establecía sus bases en 1838 con la creación del Instituto Histórico y Geográfico Brasileño (IHGB), la cual podía acercase o distanciarse de las lecturas sobre el pasado que predominaron durante el período de independencia, esto según fuese el caso de la narrativa histórica que se quisiera construir.

Por último, para los interesados en el tema de la independencia de Brasil, Escribiendo la historia del futuro es un texto novedoso, con un trabajo riguroso y bien logrado en los objetivos propuestos por la autora. Es una excelente contribución para comprender y entender la formación del Estado y la nación brasileños. Además, es una obra que nos invita a reflexionar el tema para otros espacios y tiempos, por ejemplo: ¿cuáles fueron las lecturas que hicieron los actores políticos en el mundo angloamericano e hispanoamericano respecto a sus narrativas históricas del pasado y sus pronósticos para el futuro? No sobra decir que ya hay trabajos que apuntan en esa dirección, pero aún al tema no se le ha dado la debida importancia, como se observa aquí en la obra reseñada.

Óscar Javier Castro Universidad de São Paulo / CNPq

Mercedes Prieto, comp. El Programa Indigenista Andino, 1951-1973. LAS MUJERES EN LOS ENSAMBLES ESTATALES DEL DESARROLLO. Quito: FLACSO Ecuador / Instituto de Estudios Peruanos, 2017, 336 Pp.

DOI: http: / / dx.doi.org/10.29078/rp.v0i47.681

Este libro, compilado por Mercedes Prieto, es el resultado de un trabajo interdisciplinario, "colaborativo y regional" que tiene por objetivo dar cuenta de la especificidad de "las relaciones del Programa con las mujeres indígenas y con los modos de formación de los estados andinos" (1), sin perder de vista las dimensiones regional y global. El libro tiene seis capítulos: el primero explica y problematiza, a modo de contexto, la emergencia, el alcance y las modalidades de intervención del Programa, mientras que el último, a modo de cierre, aporta con innovadoras reflexiones conceptuales respecto al vínculo entre el par "integración-desarrollo", el Estado y el género en las naciones andinas. Los cuatro restantes presentan estudios de caso en las zonas rurales de altura de Bolivia, Ecuador, Perú y Chile, en donde el diálogo entre la historia, la sociología y la antropología permite una comprensión integral de la problemática. ${ }^{1}$ Cada uno de ellos refleja el uso riguroso de dispositi- 